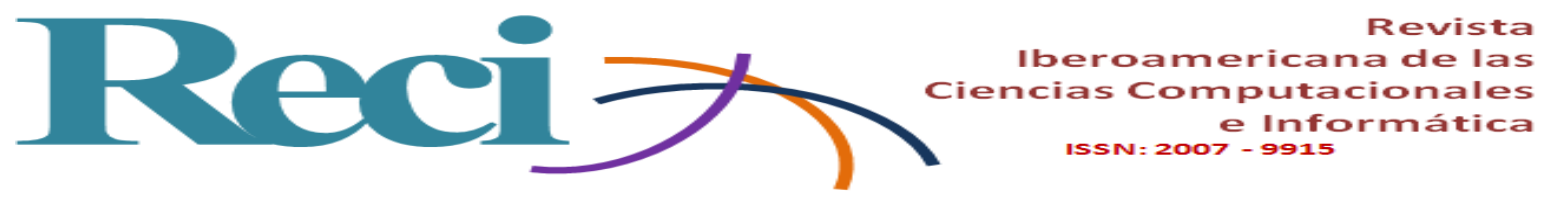

https://doi.org/10.23913/reci.v9i18.100

Artículos científicos

\title{
Propuesta de temario en un curso en cloud computing basado en competencias para la ESCOM-IPN
}

Agenda proposal in a course in Cloud Computing based on competencies for

the ESCOM-IPN

Proposta de plano de estudos em curso de computação em nuvem com base em competências para o ESCOM-IPN

Ukranio Coronilla Contreras

Instituto Politécnico Nacional, Escuela Superior de Cómputo, México

ukraniocc@yahoo.com

https://orcid.org/0000-0003-4466-9219

Virginia Medina Mejía

Instituto Politécnico Nacional, Escuela Superior de Cómputo, México

vmedinamejia@yahoo.com.mx

http://orcid.org/0000-0002-9152-7419

Gisela González Albarrán

Instituto Politécnico Nacional, Escuela Superior de Cómputo, México

gisela6543210@yahoo.com.mx

https://orcid.org/0000-0001-8086-6549 


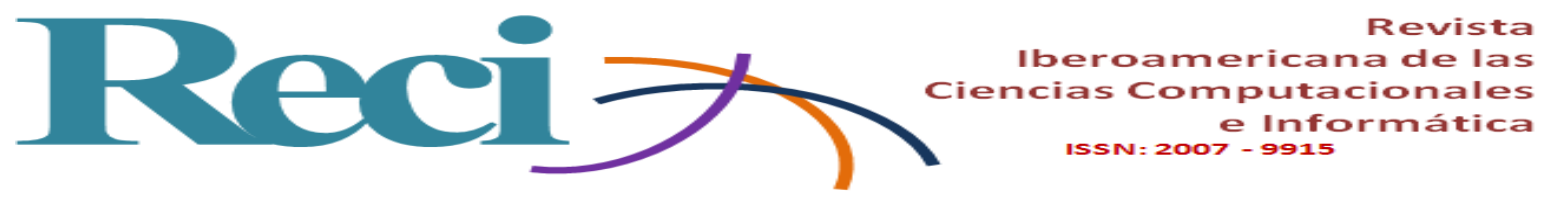

\section{Resumen}

En el momento actual, cuando se gesta la cuarta revolución industrial, es indispensable para las carreras como la ingeniería en computación y afines acoplar los contenidos curriculares de los planes de estudio para estar acordes con su consecución. Por ello, el objetivo del presente trabajo consistió en encontrar los contenidos más relevantes para un curso de cloud computing, en términos de las propuestas generadas por los más recientes estudios sobre el tema, con objeto de brindar al estudiantado las competencias que coadyuven de maneras convenientes a su desarrollo profesional en el área.

Se analizaron distintas propuestas de temarios publicadas en artículos de investigación, y con base en su impacto y en las competencias que requiere el mercado, se seleccionaron los temas que conformaron la presente propuesta. Fueron notorios los distintos enfoques que presentaron cada una de las propuestas investigadas, sin embargo, en el temario propuesto se ha conjuntado lo más relevante de todas ellas.

Palabras clave: cloud computing, cómputo distribuido, educación en ciencias de la computación.

\section{Abstract}

At the present time where the fourth industrial revolution is brewing, it is essential for careers such as computer engineering and the like; coupling the curricular contents of the study plans to be consistent with their achievement.

The objective of this work is to find the most relevant contents for a Cloud Computing course, in terms of the proposals generated by the most recent studies on the subject, in order to provide students with the competencies that contribute in convenient ways to their professional development In the area.

Different proposals for syllabi published in research articles were analyzed and based on their impact on the competences required by the market, the topics that made up this proposal were selected.

The different approaches presented by each of the investigated proposals were notorious, however, the most relevant of all of them has been brought together in the proposed agenda.

Keywords: cloud computing, distributed computing, computer science education. 


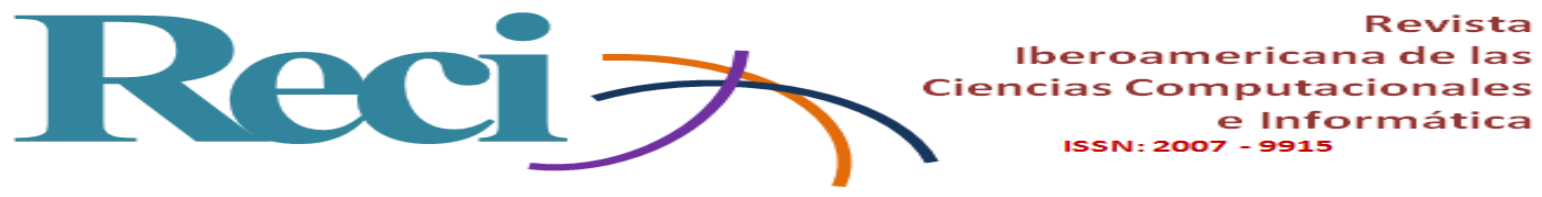

\section{Resumo}

Atualmente, quando a quarta revolução industrial está se formando, é essencial para carreiras como engenharia da computação e afins unir os conteúdos curriculares dos planos de estudo para serem consistentes com sua realização. Assim, o objetivo deste trabalho foi encontrar os conteúdos mais relevantes para um curso de computação em nuvem, no que se refere às propostas geradas pelos estudos mais recentes sobre o assunto, de forma a dotar os alunos de competências que contribuam de forma conveniente. ao seu desenvolvimento profissional na área.

Foram analisadas diferentes propostas de programas publicados em artigos de pesquisa e, com base no seu impacto e nas competências exigidas pelo mercado, foram selecionados os temas que compunham esta proposta. Foram notórias as diferentes abordagens apresentadas por cada uma das propostas investigadas, porém, as mais relevantes de todas foram reunidas na agenda proposta.

Palavras-chave: computação em nuvem, computação distribuída, ensino de ciências da computação.

Fecha Recepción: Enero 2020

Fecha Aceptación: Julio 2020

\section{Introducción}

Una tarea fundamental de toda institución educativa en la preparación de sus educandos es proponer un plan académico que responda a las necesidades de la sociedad actual, la cual empieza a transitar por una nueva etapa conocida como cuarta revolución industrial o industria 4.0. Esta se caracteriza por el desarrollo de importantes avances tecnológicos en las áreas de inteligencia artificial, procesamiento masivo de datos e interconexión ingente de dispositivos y sistemas de cómputo que fungen como proveedores de información digital.

Esta nueva revolución tecnológica, igual que ha sucedido con sus predecesoras, promete agilizar los procesos productivos, lo cual repercutirá en mejoras económicas y sociales, de ahí que sea necesario revisar y actualizar los programas de las distintas asignaturas que componen las carreras ofertadas. 


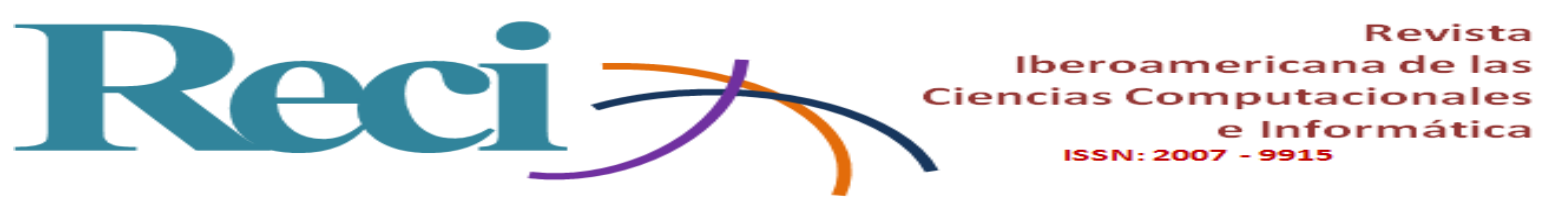

Tal es el caso del cómputo en la nube o cloud computing (CC), componente tecnológico que permite la recolección de datos, su procesamiento masivo y su distribución e intercambio entre los distintos dispositivos interconectados.

\section{Planteamiento del problema}

Para actualizar los temas que componen la asignatura obligatoria de Sistemas Distribuidos impartida en el séptimo semestre de la carrera de ingeniería en Sistemas Computacionales de la Escuela Superior de Cómputo del Instituto Politécnico Nacional (México) sin modificar el plan de estudios existente, nos proponemos encontrar los contenidos más relevantes en términos de las propuestas generadas por los más recientes estudios sobre el tema para brindar al estudiantado las competencias que coadyuven a su desarrollo profesional en esa área.

\section{Antecedentes}

Desde 1982 en México existe una Asociación Nacional de Instituciones de Educación en Tecnologías de Información (Aniei), la cual tiene, entre otras funciones, orientar y recomendar a las instituciones de educación superior en el área de informática y computación sobre la pertinencia de los temas en los planes de estudio para formar profesionistas competentes que coadyuven al desarrollo del país.

La Aniei - en una reciente publicación sobre los perfiles profesionales en TI (Sanchez et al., 2019) — reconoce que existe una tendencia hacia el cómputo en la nube debido a que diversas tecnologías como la inteligencia artificial, big data e internet de las cosas necesitan de ese servicio como una plataforma accesible para su implementación.

Tradicionalmente, los servicios de cómputo y su consumo se habían realizado mediante aplicaciones distribuidas diseñadas ad hoc; sin embargo, el cómputo en la nube expone un cambio de paradigma que obliga a revisar los cursos relacionados, como es el presente caso.

El cómputo en la nube ofrece grandes ventajas tecnológicas, entre las cuales sobresale el bajo costo asociado con las soluciones implementadas en TI, puesto que en lugar de adquirir una costosa infraestructura de equipos y licencias, solo se renta la necesaria mientras se paga por los recursos ocupados bajo demanda. El impacto económico también es creciente, 


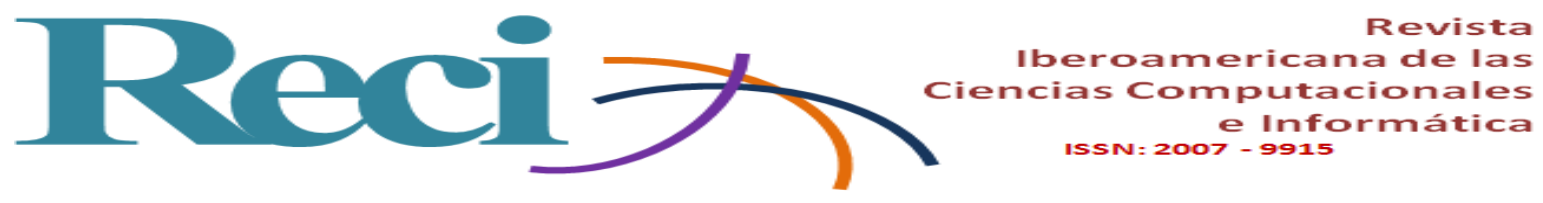

pues en un estudio realizado por Gartner se pronostican ingresos derivados del cómputo en la nube por 302000 millones de dólares.

Estadísticas reportadas en algunos portales (p. ej., Galov, 4 de diciembre de 2020) indican que proveer acceso a los datos desde cualquier ubicación es la principal razón por la que las empresas adoptan el cómputo en la nube. Asimismo, se indica que aproximadamente un tercio del presupuesto que las empresas destinan a TI son para servicios en la nube, mientras que los expertos indican que en 201960 \% de la carga de trabajo se ejecutó en la nube. Esto último incide en una optimización en el uso de energía y una reducción en los gastos para la adquisición de hardware, lo que disminuye la huella de carbono y permite a las empresas pequeñas y medianas la adquisición de tecnología.

No obstante, a pesar de la importancia del tema del cómputo en la nube, en la actualidad existen serias deficiencias en la preparación de profesionistas capacitados en el área. Como comentan Foster et al. (2018), no solo en el Reino Unido existe escasez de competencias en el área, sino que también en una encuesta de investigación a nivel global entre académicos e industrias se indica que las organizaciones están teniendo pérdidas debido a una carencia de expertos. Por ende, se requiere un enfoque práctico en el temario para adquirir las experiencias necesarias para el buen desempeño del profesionista egresado.

\section{Cómputo en la nube}

Para la definición de cómputo en la nube utilizaremos la que ofrece el National Institute of Standards and Technology (NIST):

La computación en la nube es un modelo para permitir el acceso sobre demanda por red, de manera conveniente y ubicua a un grupo compartido de recursos informáticos configurables (por ejemplo, redes, servidores, almacenamiento, aplicaciones y servicios) que se pueden proveer y liberar rápidamente con un mínimo esfuerzo de administración o interacción del proveedor de servicios (Mell y Grance, 2011, p. 2).

Dicho modelo se compone de tres servicios conocidos como Software as a service (SaaS), Platform as a service (PaaS), e Infrastructure as a service (IaaS). En SaaS el servicio incluye la ejecución de software para el usuario con la infraestructura del proveedor (p. ej., el servicio de correo Gmail, el editor de textos Google Docs y el servicio de almacenamiento en línea Dropbox, entre otros). En PaaS el proveedor del servicio ofrece una plataforma sobre 


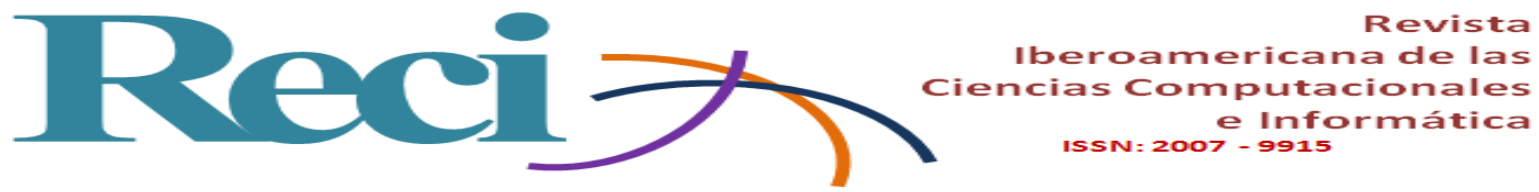

la cual se ejecutan las aplicaciones desarrolladas con la API y las bibliotecas del mismo proveedor, de manera que es posible crear aplicaciones como las que se realizan con web .net, ejecutándose en instancias de Windows cloud Azure. Por último, en IaaS el servicio consiste en infraestructura de hardware virtual, como las máquinas virtuales, el espacio de almacenamiento y las redes de conexión, los cuales pueden ser configurados y ejecutados por los usuarios.

Existen también tres modelos básicos de acceso a la nube: el público, el privado y el hibrido. En el caso del público, un proveedor brinda la infraestructura y los servicios, los cuales están disponibles mediante suscripción para cualquier usuario interesado vía internet. En el modelo privado, la nube está disponible a usuarios dentro de una compañía gracias a su propio centro de datos. Finalmente, en las nubes híbridas tenemos una mezcla de nubes privadas y públicas, las cuales se comienzan a utilizar cuando las capacidades de la nube privada son insuficientes.

El cómputo en la nube es producto de décadas de investigación y desarrollo en distintas tecnologías que incluyen la virtualización, las redes de cómputo y los sistemas de cómputo distribuidos, pasando por el desarrollo de clústeres y el cómputo de malla (grids), como se muestra en la figura 1.

Figura 1. Evolución histórica del cómputo distribuido

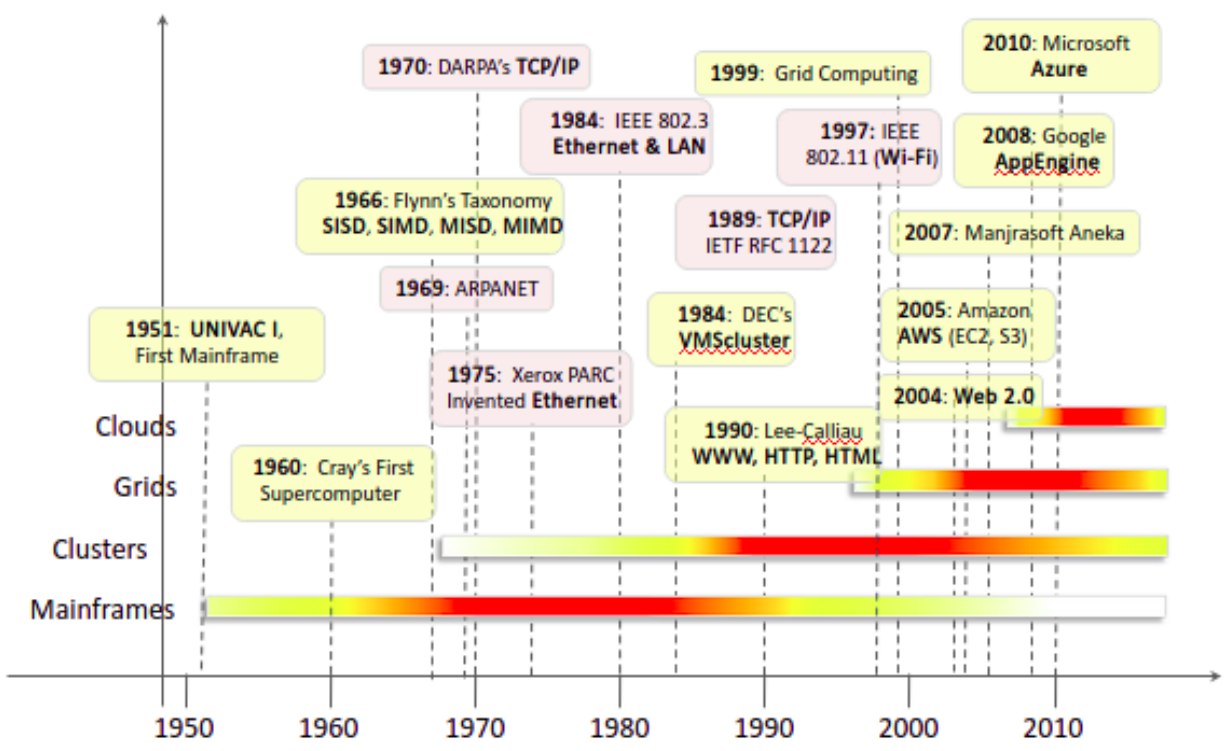

Fuente: Buyya, Vecchiola y Selvi (2013) 


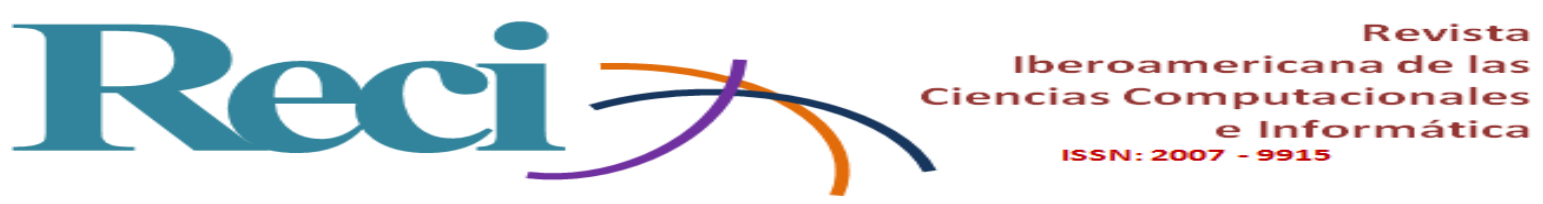

En la figura 1 podemos observar que uno de los componentes que impulsan el cómputo en la nube es la aparición de la Web 2.0 en 2004. A partir de entonces comienzan a aparecer proveedores de servicios en la nube como Amazon en 2005, Google en 2008 y Microsoft Azure en 2010.

\section{Casos de estudio}

La enseñanza del cómputo en la nube lleva aparejadas varias dificultades, como por ejemplo la diversidad de conocimientos previos que necesitan dominar los instructores. En tal sentido, es posible afirmar que el cómputo en la nube es un área multidisciplinaria debido a que se encuentran involucrados conceptos de diversos campos, como los sistemas operativos, las bases de datos, las redes de cómputo y los sistemas distribuidos. Por este motivo, un curso de cómputo en la nube se estudia en los niveles avanzados de las carreras que no se especializan en dicho paradigma, como es el presente caso.

A continuación, expondremos los antecedentes revisados que incluyen los temas propuestos, así como las competencias asociadas más relevantes; posteriormente, realizaremos la selección de los temas más convenientes a partir de su impacto en las necesidades del creciente mercado. Hemos dejado expresadas las competencias generales para cada caso de estudio con la intención de que cada centro educativo elabore sus competencias específicas basándose en los propios criterios que defina el cuerpo colegiado de profesores.

En la investigación de Foster et al. (2018) se indica que las tecnologías de nube modernas incluyen diversas herramientas que van cambiando constantemente debido a la competencia que existe entre los proveedores de servicios de nube, los cuales no solo generan nuevos diseños e implementación de conceptos, sino que también producen cambios en las interfaces gráficas y líneas de comandos. Esto obliga a los instructores a tener que actualizarse con frecuencia con curvas de aprendizaje pronunciadas, lo cual puede ocasionar renuencia por parte de los implicados. Esto, además, ocasiona que el material gráfico elaborado y los libros de texto vayan perdiendo vigencia semestre a semestre, lo que hace complicado mantener una bibliografía actualizada.

Otro elemento de dificultad a considerar es la cantidad y diversidad de proveedores de servicios para la nube, pues cada uno ofrece distintas herramientas y tecnologías, así como 


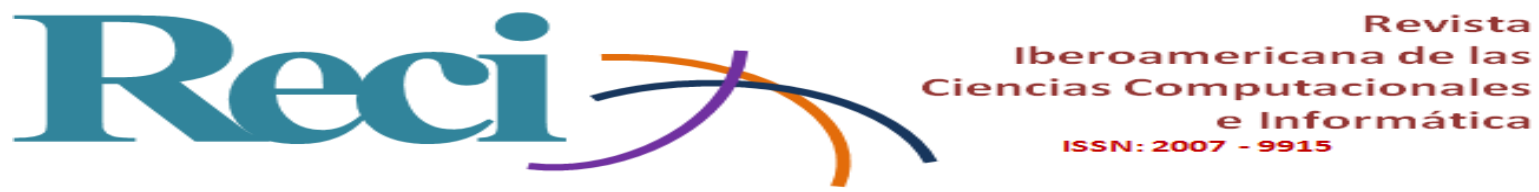

interfaces propias. En Foster et al. (2018) recomiendan utilizar más de un proveedor de servicios de nube para centrarse en los conceptos y evitar la mecanización. Algunos de esos proveedores y páginas asociadas se enlistan en la figura 2.

Figura 2. Proveedores de servicios en la nube AWS: https://aws.amazon.com/education/awseducate

Azure: https:///azure.microsoft.com/en-us/education

GCP: http://cloud.8oogle.com/edu

IBM: https://developer.ibm.com/academic/docs/cloud-offer

Oracle: https://cloud.oracle.com/home

Openstack: https://www.openstack.org

VMware: https://www.vmware.com

Heroku: https://www.heroku.com/students

ChameleonCloud: https://www.chameleoncloud.org

CloudLab: https://cloudlab.us

Geni: http://www.beni.net

Federation for Fire: $\underline{\text { https: }} / /$ www.fed4fire.eu

PlanetLab: https://www.planet-lab.org

Alibaba Cloud https://www.alibabacloud.com/

DigitalOcean https: //www.digitalocean.com/.

Fuente: Foster et al. (2018)

En el mismo trabajo, se proponen las siguientes áreas de conocimiento como las más relevantes:

- Conceptos fundamentales de la nube.

- Abstracciones computacionales en la nube.

- Recursos de almacenamiento en la nube.

- Recursos de redes de interconexión en la nube.

- Elasticidad y escalabilidad en la nube.

- Tolerancia a fallas, resiliencia y confiabilidad.

- Desarrollo, monitoreo y mantenimiento en la nube.

- Orquestación en la nube.

- Desarrollo de software usando API de la nube.

- Modelos de programación en la nube y Frameworks. 


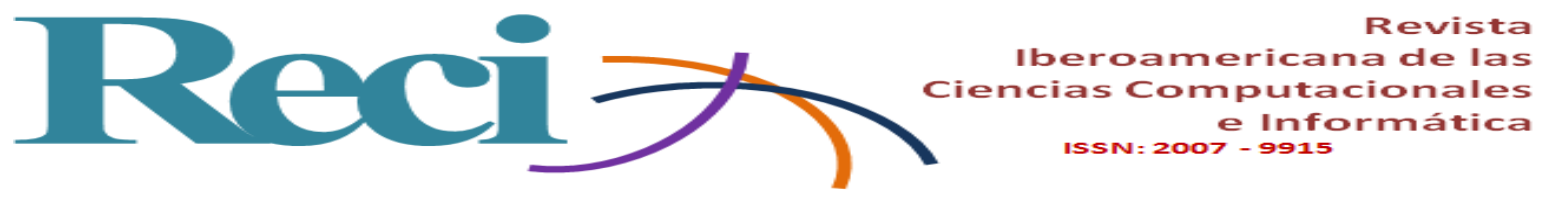

- Arquitectura orientada a servicios.

- Seguridad, privacidad, políticas y ética en la nube.

- Internet de las cosas.

- Inteligencia artificial y aprendizaje automático basado en la nube.

Como es posible observar, en los dos últimos temas propuestos el cómputo en la nube es una tecnología clave que sirve como plataforma de apoyo para otras áreas importantes de las TI, como la inteligencia artificial, big data e internet de las cosas.

Por otra parte, en el trabajo de Chen et al. (2012) se propone un curso de cómputo en la nube básico para usar tecnologías que apoyan a distintas materias que conforman un plan de estudio destinado al procesamiento de datos de gran escala, su análisis y visualización. Un mapa con las tecnologías y los cursos asociados se muestra en la figura 3.

Figura 3. Mapeo de cursos y tecnologías cloud

\begin{tabular}{|c|c|c|c|c|}
\hline & & Cloud Component & $\begin{array}{l}\text { Course } \\
\text { Components }\end{array}$ & Courses \\
\hline & & Hosting Websites & Data warehouse & $\begin{array}{l}\text { INFS3200 Advanced } \\
\text { Database Systems }\end{array}$ \\
\hline & & on Cloud & Web design & INFS3202 Web \\
\hline \multicolumn{2}{|c|}{ Cloud Technologies } & Cloud data & $\begin{array}{l}\text { scripting } \\
\text { language }\end{array}$ & Information Systems \\
\hline \multirow{3}{*}{$\begin{array}{l}\text { Cloud } \\
\text { Services }\end{array}$} & Amazon & warehouse tool & Visual Studio & INFS3204 Service \\
\hline & EC2 & Cloud Web Service & Web service & Oriented Architectures \\
\hline & $\begin{array}{l}\text { Microsoft } \\
\text { Azure }\end{array}$ & $\begin{array}{l}\text { MapReduce } \\
\text { K-means }\end{array}$ & $\begin{array}{l}\text { - K-means } \\
\text { - Matlab }\end{array}$ & $\begin{array}{l}\text { COSC3500 High- } \\
\text { Performance } \\
\text { Computing }\end{array}$ \\
\hline \multirow{3}{*}{$\begin{array}{l}\text { Distributed } \\
\text { Computing } \\
\text { Framework } \\
\text { (MapReduce- } \\
\text { based) }\end{array}$} & Hadoop & Scientific & \multirow{2}{*}{$\begin{array}{ll}- & \text { Scientific } \\
\text { Visualization } \\
\text { - } \quad \text { Matlab }\end{array}$} & \multirow{2}{*}{$\begin{array}{l}\text { COSC3000 } \\
\text { Visualisation, Computer } \\
\text { Graphics and Data } \\
\text { Analysis }\end{array}$} \\
\hline & Mahout & $\begin{array}{l}\text { computing using } \\
\text { MapReduce }\end{array}$ & & \\
\hline & Hive & \multirow{3}{*}{$\begin{array}{l}\text { Matlab parallel } \\
\text { computing on } \\
\text { cloud }(X)\end{array}$} & \multirow{3}{*}{$\begin{array}{l}\text { Scientific } \\
\text { Computing } \\
\text { Matlab }\end{array}$} & \multirow{2}{*}{$\begin{array}{l}\text { MATH3201 Scientific } \\
\text { computing: advanced } \\
\text { techniques and } \\
\text { applications }\end{array}$} \\
\hline & & & & \\
\hline & & & & $\begin{array}{l}\text { MATH4201 Applications } \\
\text { of scientific computing }\end{array}$ \\
\hline
\end{tabular}

Fuente: Chen et al. (2012)

En este caso, es posible observar que además de las tecnologías de servicios de nube comerciales como Amazon y Azure, se emplean plataformas como Hadoop que son de código abierto gratuito y que permiten implementar con rapidez un clúster con computadoras de propósito general para procesar grandes cantidades de datos en poco tiempo manteniendo la flexibilidad, la tolerancia a fallos y la escalabilidad. En el mismo trabajo de Chen et al. (2012) se describe la implementación de un pequeño clúster con Hadoop instalado en cuatro 


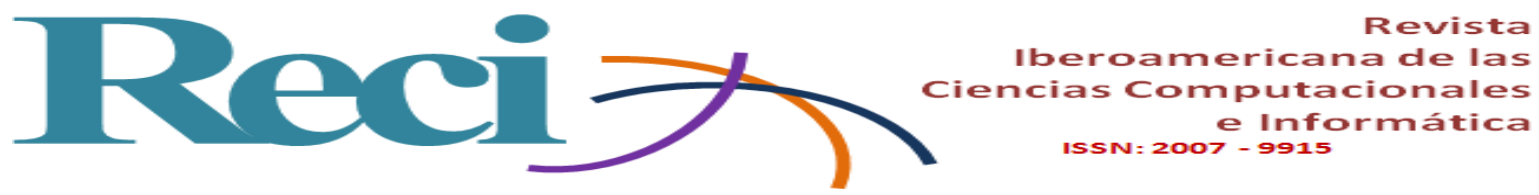

computadoras, las cuales mediante técnicas MapReduce procesan un conjunto de datos obtenidos de la biblioteca de medicina nacional de Estados Unidos Medline, con un tamaño de $50.7 \mathrm{~GB}$ en 40 minutos.

MapReduce es una técnica desarrollada por Google para procesar grandes cantidades de datos de manera eficiente, la cual la hace pertinente para su uso en big data, y se construye sobre un sistema de archivos distribuido. En vista de su relevancia, el proveedor de servicios de nube AWS desarrolló una implementación de MapReduce conocida como Elastic Map Reduce. Aunque no se reporta en el trabajo, es posible inferir que si se dispone de un laboratorio o si los alumnos cuentan con computadoras personales, es posible realizar procesamiento de grandes cantidades de datos sin tener que pagar a un proveedor de servicios de nube.

Asimismo, en el trabajo Rodríguez et al. (2014) se proponen los siguientes temas como fundamentales en un curso de cómputo en la nube:

- Conceptos introductorios.

- Clasificación de servicios: SaaS, PaaS, IaaS.

- Modelos de despliegue: público, privado e híbrido.

- Virtualización y data centers.

- Clústeres y arquitecturas de HPC.

- Base de Datos NoSQL y big data.

- MapReduce.

- Programación del cloud y ambientes de software.

- Ambientes de software emergentes: Open Source, Eucalyptus y Nimbus.

- Ciclo de vida y metodología para cloud computing.

- Prácticas que pueden ser sobre PaaS o SaaS.

- Aspectos legales de cloud computing, fundamentalmente SLA para cloud (Service Level Agreement).

Con un tiempo total asignado de 23 horas tanto para la parte teórica como para algunas prácticas asociadas, en este caso se pensó en un conjunto de temas mínimos que sirvieran de apoyo a distintas asignaturas del plan de estudios. Entre las competencias fundamentales sobresalen que el alumno tenga conocimientos sobre los fundamentos del cómputo en la 


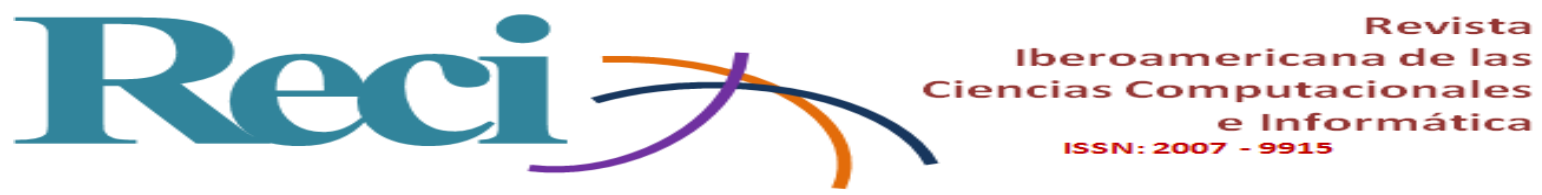

nube, las tecnologías de apoyo asociadas, sus ventajas y limitaciones, las técnicas de creación y uso de la nube y los fundamentos de MapReduce como modelo de programación.

En el trabajo de Border (2013) se enfatiza que la fortaleza de desarrollar aplicaciones en la nube no viene de las implementaciones en un solo servidor ni de trabajar con una sola tecnología, sino de implementar soluciones que incluyan múltiples servicios en distintos servidores ubicados en centros de datos de todo el mundo. Por ello, el autor recalca la importancia de que los estudiantes tengan los conocimientos necesarios para administrar la forma en que interactúan los distintos servidores. Desde esta perspectiva, el autor ubica las siguientes tecnologías y servicios como fundamentales para revisarse en un curso de cómputo en la nube:

- Firewalls, lo que incluye tener conocimientos sobre el uso de puertos y protocolos para supervisar el tráfico.

- Direccionamiento y subneteo con el propósito de configurar puntos únicos de ingreso y egreso de datos.

- Cifrado para dar solución a los temas de seguridad de los datos que viajan hacia y desde la nube.

- Scripting para facilitar el uso de lenguajes como Java, .NET, y PHP adicionales a la interfaz gráfica.

- Arquitecturas de almacenamiento que permitan conocer las formas de replicación, pues esta es una de las razones más importantes para usar la nube.

- Virtualización y las implicaciones que esta tiene.

- Segregación de servicios, lo que implica temas como la distribución de tráfico y el almacenamiento de imágenes de sistema para su migración.

- Caracterización de la carga de trabajo en los servidores para prevenir el efecto barrapunto.

- Identidad federada para la administración interempresarial de los recursos en la nube.

- Balanceo de carga y su administración en la nube.

- Content delivery network para permitir a los usuarios acceder a contenidos en servidores más cercanos. 


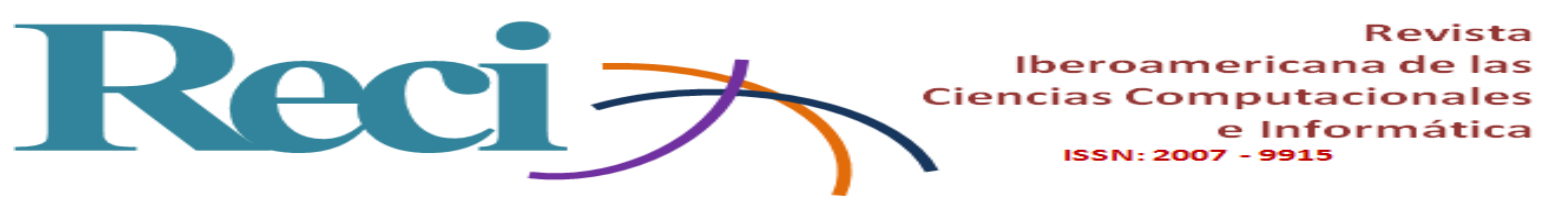

Como se puede inferir, en este caso existen temas y competencias que requieren de los vistos anteriormente, y que se centran en la administración de los recursos que conforman las distintas tecnologías que brindan los proveedores de servicios en la nube.

En el estudio de Breivoid y Crnkovic (2014) se menciona que, en el área de cómputo en la nube, los estudiantes se sienten más motivados a aprender cuando observan cómo se aplican los conceptos en el mundo real. En otras palabras, además de mostrarles los casos donde ellos han interactuado con aplicaciones en la nube muy conocidas como las redes sociales, videoconferencias, datos almacenados en la nube, etc., deben familiarizarse con áreas de negocios y de nivel empresarial donde se incorpora el cómputo en la nube, por ejemplo:

- En análisis de datos y cómputo donde se requieren grandes capacidades de procesamiento en paralelo, y donde es posible el uso de herramientas como el almacenamiento y cómputo elástico para poder llevarlo a cabo.

- En el desarrollo y pruebas de software donde es posible ocupar la infraestructura de cómputo en la nube para modelar de manera realista su puesta en práctica.

- La colaboración global interempresarial para compartir información y datos además del desarrollo de procesos y herramientas, que se vuelven posibles gracias al cómputo en la nube.

Los autores recomiendan la introducción de estos ejemplos con objeto de ayudar al estudiante a comprender la naturaleza abstracta del cómputo en la nube. Entre las áreas de conocimiento clave que Breivoid y Crnkovic (2014) mencionan están las siguientes:

- Arquitectura para la nube, que incluye a aquellas que reúnen los requerimientos del sistema como disponibilidad, interoperabilidad, desempeño, mantenibilidad, rendimiento, confiabilidad y escalabilidad.

- Diseño para la resiliencia, que implica la inclusión de elementos como redundancia, tolerancia a fallos y técnicas asociadas como respaldos dinámicos y recuperación.

- Seguridad en la nube, lo cual implica conocer las ventajas y desventajas de tener un proveedor de servicios en la nube, sus amenazas de seguridad y posibles soluciones.

- Migración a la nube, lo que incluye la capacidad de evaluar los costos y decisiones de diseño necesarias para que una organización pueda llevar dicha tarea. 


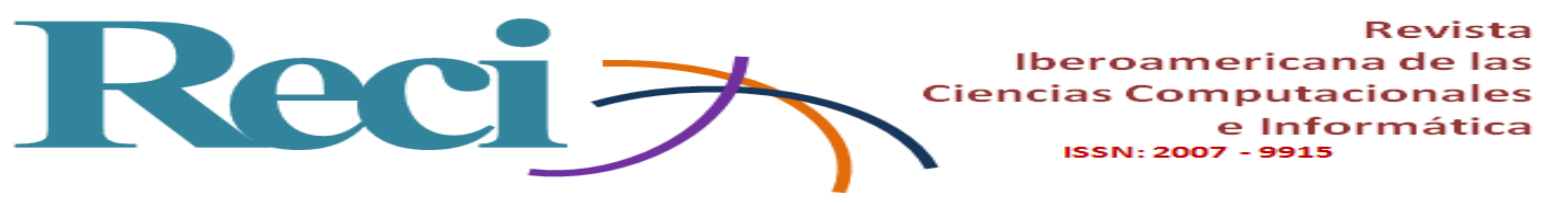

\section{Discusión}

Un modelo de la arquitectura de cómputo en la nube se expone en el trabajo de Demchenko et al. (2013), el cual cuenta con seis capas (figura 4). En ese modelo podemos observar que, en los casos hasta ahora revisados, los temas que se tratan corresponden a las capas tres, cuatro y cinco. Es decir, todo lo que tiene que ver con la orquestación de los servicios virtuales, los servicios de la nube (infraestructura, plataformas, aplicaciones, software) y los servicios de acceso/entrega. Visto desde esta perspectiva, tiene sentido exponer los temas de acuerdo con dicha agrupación para revisar de manera conjunta los que se encuentran en la misma capa.

A continuación, se muestra la propuesta de temario para un curso semestral de cómputo en la nube, teniendo en cuenta que las competencias en temas iniciales son fundamentales para lograr las competencias en temas subsecuentes. Asimismo, y por las razones expuestas en los casos de estudio, se ha evitado especificar al proveedor de servicios de nube, lo cual dependerá de la decisión al profesor o grupo de profesores encargados del curso, aunque se recomienda utilizar dos o más proveedores de servicios de nube en cada práctica. 


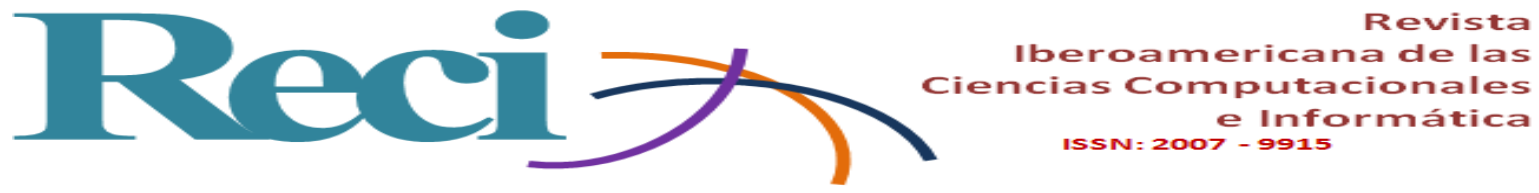

Figura 4. Modelo multicapa referencia de servicios de nube

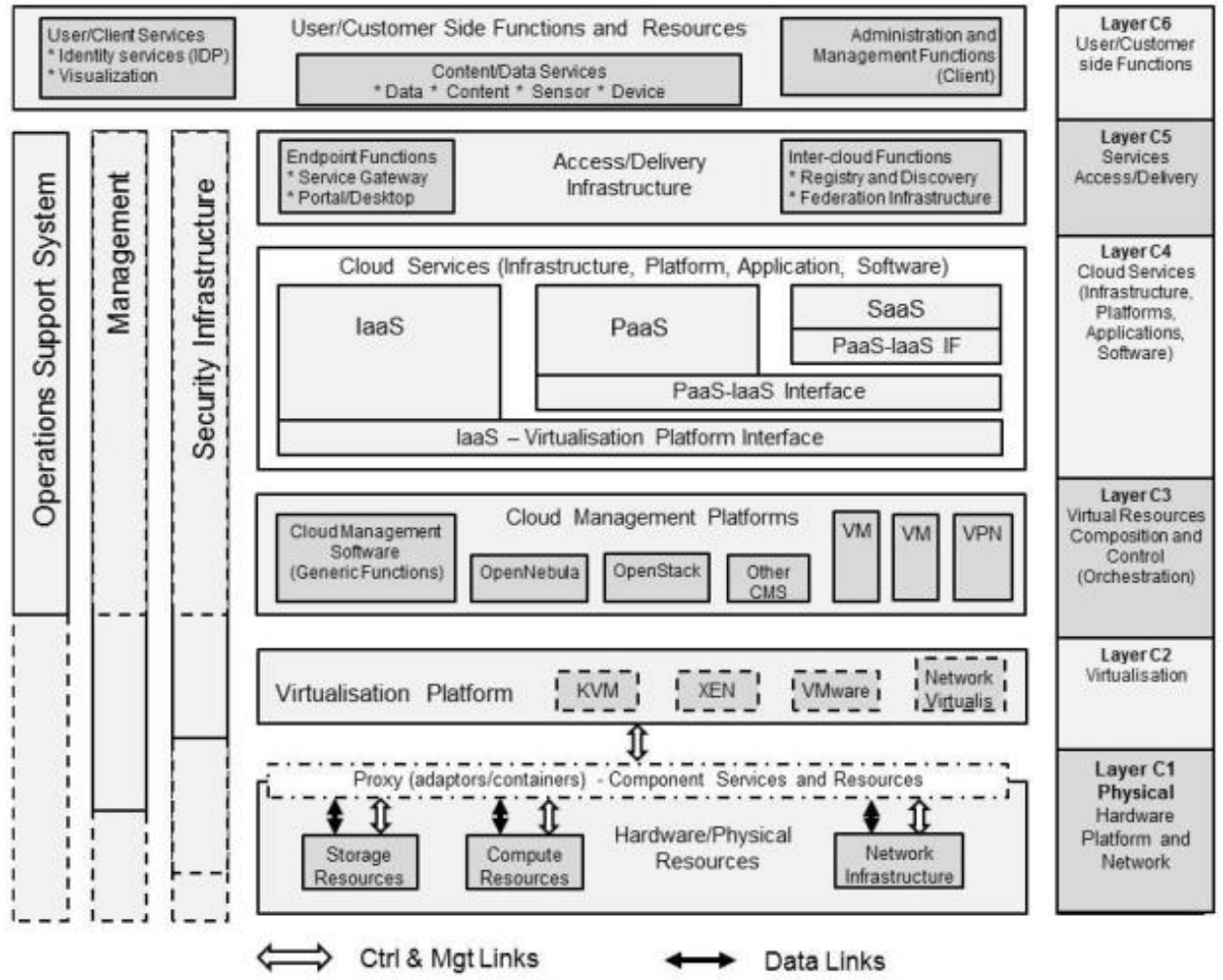

Fuente: Demchenko et al. (2013)

1. Introducción y antecedentes históricos del cómputo en la nube.

2. Virtualización y data centers.

3. Ejemplos de aplicaciones de cómputo en la nube para

3.1. Análisis de datos.

3.2. Desarrollo y pruebas de software.

3.3. Ambientes colaborativos.

4. Procesamiento distribuido basado en técnicas MapReduce.

5. Conceptos introductorios de cómputo en la nube

5.1. Clasificación de servicios (SaaS, PaaS, IaaS).

5.2. Modelos de despliegue: público, privado e híbrido.

5.3. Disponibilidad, interoperabilidad, desempeño, mantenibilidad, rendimiento, confiabilidad y escalabilidad.

6. Programación del cloud y ambientes de software para:

6.1. Virtualización y sus implicaciones.

6.2. Arquitecturas de almacenamiento y formas de replicación. 


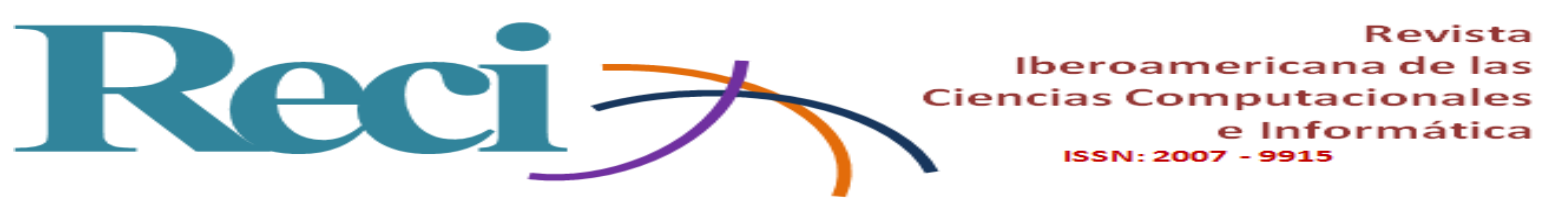

6.3. Elasticidad y escalabilidad.

6.4. Recursos de redes de interconexión.

6.5. Tolerancia a fallas, resiliencia y confiabilidad.

7. Programación de servicios en la nube que incorporan:

7.1. Seguridad, cifrado, Firewalls, direccionamiento y subneteo.

7.2. Balanceo de carga y su administración.

7.3. Content delivery network.

8. Aspectos legales de cloud computing

8.1. SLA (Service Level Agreement) para cloud

\section{Conclusiones}

Al término de la presente investigación hemos podido constatar, por los artículos revisados, una enorme inquietud de la comunidad académica por encontrar los temas que mejor se ajunten al objetivo común de brindar las mejores herramientas a los estudiantes para enfrentar con éxito las áreas de desarrollo asociadas con esta cuarta revolución industrial.

Definitivamente, uno de los problemas más complicados a los que se enfrentan los educadores en el área de cómputo en la nube tiene que ver con la velocidad con que cambian y se actualizan las herramientas, así como la gran variedad de proveedores, pues cada uno de ellos ofrece distintas interfaces y procedimientos. Estos cambios son impulsados por las exigencias del mercado y la competencia entre los proveedores de servicios de nube. Esto provoca a su vez que no exista un consenso sobre cuál es el mejor proveedor de servicios en la nube en términos pedagógicos, es decir, no existe un proveedor que cubra todas las herramientas tecnológicas disponibles ni un temario fijo que se encuentre mejor adaptado a las múltiples necesidades del mercado.

Es posible observar al menos tres tendencias en cuanto a la forma de enseñar el cómputo en la nube a alumnos de licenciatura: la primera es la de agrupar un núcleo básico de conocimientos que permita formar las competencias necesarias para abordar otros temas que forman parte de esta revolución informática, como lo son la inteligencia artificial, big data e internet de las cosas. La segunda tiende a abordar los temas relacionados con el cómputo masivo de datos, dada la importancia de áreas tecnológicas como la minería de datos o el big data. Y una tercera vinculada con la administración e interconexión de servicios en la nube, con objeto de brindar las características deseables de un sistema distribuido como la 


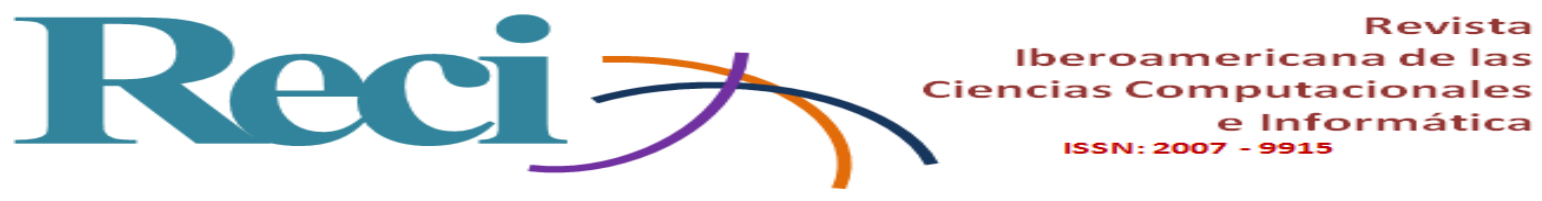

tolerancia a fallos, la replicación, la alta disponibilidad y la confiabilidad. A lo largo de este trabajo hemos tratado de conjuntar todas ellas en un único temario, el cual esperamos tenga el impacto necesario en nuestros futuros profesionistas.

\section{Futuras líneas de investigación}

Como futuras líneas de investigación están la de hacer una evaluación de entre todas las posibles proveedoras de servicios de nube, para ubicar a las más pertinentes en el apoyo del presente temario, principalmente por sus características de adaptabilidad, facilidad de uso y disponibilidad.

Otra área de investigación se centraría en encontrar los mecanismos más eficientes para incorporar los nuevos desarrollos tecnológicos provistos por las proveedoras de servicios de nube, ocupando estrategias didácticas para la educación 4.0, que permitan atenuar la sobrecarga para los docentes y alumnos en las pronunciadas curvas de aprendizaje que se requieren en este campo. 


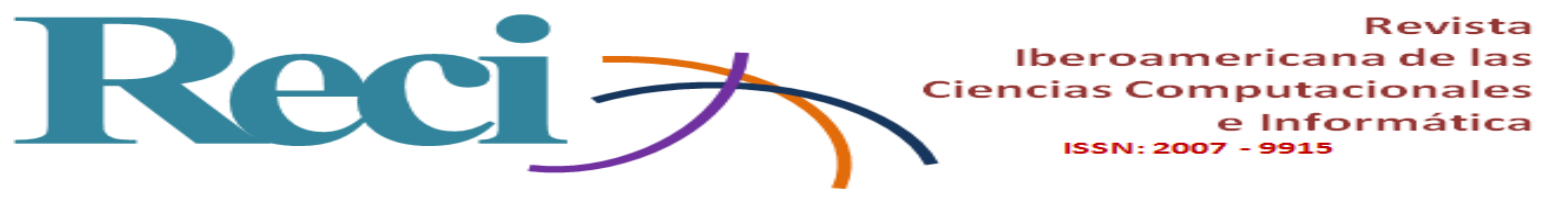

\section{Referencias}

Border, C. B. (2013). Cloud computing in the curriculum: fundamental and enabling technologies. In Proceeding of the 44th ACM technical symposium on Computer science education (pp. 147-152).

Breivold, H. P. and Crnkovic, I. (2014). Cloud Computing education strategies. In 2014 IEEE $27^{\text {th }}$ Conference on Software Engineering Education and Training (CSEE\&T) (pp. 29-38). IEEE.

Buyya, R., Vecchiola, C. and Selvi, S. T. (2013). Mastering cloud computing: foundations and applications programming. Newnes.

Chen, L., Liu, Y., Gallagher, M., Pailthorpe, B., Sadiq, S., Shen, H. T. and Li, X. (2012). Introducing cloud computing topics in curricula. Journal of Information Systems Education, 23(3).

Demchenko, Y., Bernstein, D., Belloum, A., Oprescu, A., Wlodarczyk, T. W. and De Laat, C. (2013). New instructional models for building effective curricula on cloud computing technologies and engineering. In 2013 IEEE 5th International Conference on Cloud Computing Technology and Science (vol. 2, pp. 112-119). IEEE.

Foster, D., White, L., Adams, J., Erdil, D. C., Hyman, H., Kurkovsky, S.,... and Stott, L. (2018). Cloud computing: developing contemporary computer science curriculum for a cloud-first future. In Proceedings Companion of the 23rd Annual ACM Conference on Innovation and Technology in Computer Science Education (pp. 130-147).

Galov, N. (4 de diciembre de 2020). 25 Cloud Computing Statistics in 2020 - Will AWS Domination Continue? HostingTribunal. Retrieved from https://hostingtribunal.com/blog/cloud-computing-statistics/\#gref

Mell, P. and Grance, T. (2011). The NIST definition of cloud computing.

Rodríguez, N. R., Valenzuela, A., Villafañe, D. A., Murazzo, M. A., Chávez, S. B. y Martín, A. E. (2014). Una propuesta para la incorporación de Cloud Computing en la currícula de Grado. Revista Iberoamericana de Tecnología en Educación y Educación en Tecnología, (12), 37-43.

Sánchez, L., Aguas, N., Cortés, J. R., Gaona, A. R., Rodríguez-Abitia, G. y Rodríguez, F. J. A. (2019). Nuevos perfiles profesionales en TI: caso ANIEI. Recuperado de https://aisel.aisnet.org/amcis2019/spanish_portuguese_latin_america/spanish_portu guese_latin_america/44/

\section{(C)}




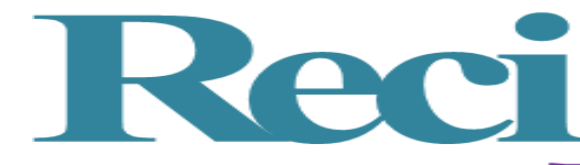

\begin{tabular}{|l|l|}
\hline Rol de Contribución & Autor (es) \\
\hline Conceptualización & Ukranio Coronilla Contreras \\
\hline Metodología & Virginia Medina Mejía \\
\hline Software & Ukranio Coronilla Contreras \\
\hline Validación & Gisela González Albarrán \\
\hline Análisis Formal & Ukranio Coronilla Contreras \\
\hline Investigación & Virginia Medina Mejía \\
\hline Recursos & Gisela González Albarrán. \\
\hline Curación de datos & Ukranio Coronilla Contreras \\
\hline $\begin{array}{l}\text { Escritura - Preparación del } \\
\text { borrador original }\end{array}$ & Ukranio Coronilla Contreras \\
\hline $\begin{array}{l}\text { Escritura - Revisión y } \\
\text { edición }\end{array}$ & Virginia Medina Mejía \\
\hline Visualización & Gisela González Albarrán \\
\hline Supervisión & Ukranio Coronilla Contreras \\
\hline Proyectos & Virginia Medina Mejía \\
\hline Adquisición de fondos & \\
\hline
\end{tabular}

